\title{
Free Radicals and Oxidative Stress
}

\section{Monica Butnariu ${ }^{1 *}$ and lonel Samfira ${ }^{2}$}

${ }^{1}$ Chemistry and Vegetal Biochemistry, Banat's University of Agricultural Sciences and Veterinary Medicine, Timisoara, Romania

${ }^{2}$ Forage Department, Banat's University of Agricultural Sciences and Veterinary Medicine, Timisoara, Romania

Free Radicals (FR) are substances that derive from incompletely oxidated compounds that have undergone partial burning and that have, in their structure, oxygen groups capable of initiating, at the surface of the cell membranes or even within the cells, aggressive oxidation reactions. FR result from both processes occurring in the body (incomplete catabolism, energy production, hepatic detoxification, etc.) and the outer environment (cigarette smoke, polluted air, foods, medicines, well/tap water, etc.) [1]. Radicals are generated within the initiation stage; they participate in a sequence of propagation reactions in which the number of radicals is constant; they are later on destroyed during the chin interruption stage. Identifying FR can be done through spin electronic resonance. The spin electronic resonance (electronic paramagnetic) method is based on the spectroscopic observation of some transitions between different energy levels depending on the electronic spin orientation. From an electric point of view, FR has a level-headed character (they generally have no charge) [2]. The presence of unpaired electrons makes these species unstable, FR being very reactive to the interaction with other molecules because they need to pair the electrons and to make up a more stable compound. Though, in some cases, the molecules need extreme conditions to make up radicals, many cell compounds can be turned into radicals under relatively soft conditions, including those specific to living organisms. The forming of FR is complicated and it can initiate a series of unpredictable reactions in the body, damaging lipids, albumens and nucleic acid.

In living cells, the forming of FR takes place mainly through such processes as the homolysis of chemical bonds, photolysis, and radiolysis, and as a result of redox reactions. In the cells, FR is made up continuously, as by-products of oxygen metabolism during the oxidative phosphorylation taking place in mitochondria. The most probable way to initiate the forming of FR in the cells is the process in which the homolysis of chemical bonds is accompanied by the forming of other bonds, which leads to a significant decrease of the process activation energy on the whole. Oxidative stress is defined as an exaggerated production of oxygenated FR, accompanied by a dislocation of anti-oxidation agents. We cannot live without oxygen, since it is essential in the functioning of energy-producing cells [3]. A body transforms and eliminates carbon dioxide $\left(\mathrm{CO}_{2}\right)$ almost entirely (98\%). Unfortunately, the rest is at the origin of some "hyper-reactive" species called FR. FR are oxidated derivatives of the electron deficit, unstable oxygen molecule, that cause dysfunctions of all body cells. The volume of oxygen contained by a single inspiration produces a billion FR. Oxygenated FR are at the origin of chain reactions; such a radical, unstable as a result of the electron deficit, captures the missing electron from a "foreign" molecule that becomes, in its turn, an unstable radical, and the process goes on and on.

\section{Free radical action mechanisms and organism defense mechanisms}

The main actions of FR in a biological system are (Figure 1) oxidation of PUFA in the cell membrane, oxidation of amino acids

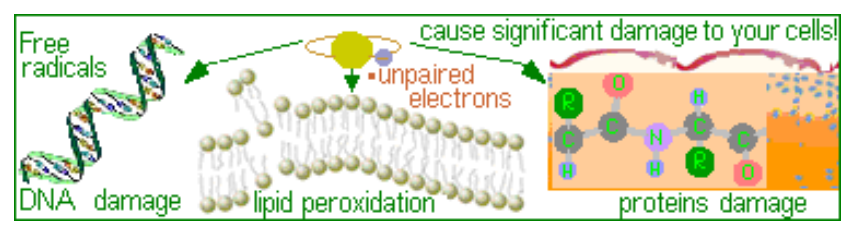

Figure 1: Reactive oxygen species damage DNA, lipids, and proteins.

in proteins, depolymerisation of hialuric acid, oxidative oxidation of DNA, modulation of the activity of nucleotide cyclase, modulation of prostaglandin activity and synthesis, etc.

The current concepts of Reactive Oxygen Species (ROS) signaling can be grouped into two action mechanisms-alterations of intracellular redox state and protein oxidative changes.

\section{Alterations of intracellular redox state}

Compared to the extra-cell environment, cytoplasm is normally maintained in reducing conditions (accompanied by the buffer redox capacity of intra-cell thiol such as GSH and TRX). These thiol systems oppose intra-cell oxidative stress reducing $\mathrm{H}_{2} \mathrm{O}_{2}$, lipid super oxides and peroxides (CAT by HRP).

\section{Protein oxidative changes}

ROS can alter the structure and function of proteins, changing the rest of amino acids, inducing protein dimerization and interacting with other metal complexes (Fe-S). Oxidative changes of amino acids in the protein functional domain can involve several pathways. The target cell components of free radical action include lipids (LDL), macromolecules with complex structure, proteins and DNA. To minimize the negative effects of ROS, organisms are endowed with very efficient antioxidant defense system. Antioxidant compounds (vitamins E and C, uric acid and $\beta$-carotene), antioxidant enzymes (SOD, CAT, HRP), and metal chelation agents (transferrin, lactoferrin and ceruloplasmin) function as an antioxidant system by bonding potential damaging metal ions. In oxidative stress, the ratio between pro-oxidants and antioxidants is in favor of the former.

The pro-oxidative status is engendered by the presence of FR, while the anti-oxidative one is due to free antiradicals; together, they make

*Corresponding author: Monica Butnariu, Ph.D. in Agricultural Sciences, Associate professor, Degree in Chemistry and Vegetal Biochemistry, 300645 Calea Aradului 119, Timis, Romania, Tel: +40-0-256-277-441; Fax: +40-0-256200-296; E-mail: monicabutnariu@yahoo.com / monica_butnariu@usab-tm.ro

Received April 06, 2012; Accepted April 10, 2012; Published April 12, 2012

Citation: Butnariu M, Samfira I (2012) Free Radicals and Oxidative Stress. J Bioequiv Availab 4: iv-vi. doi:10.4172/jbb.10000e13

Copyright: (c) 2012 Butnariu M, et al. This is an open-access article distributed under the terms of the Creative Commons Attribution License, which permits unrestricted use, distribution, and reproduction in any medium, provided the original author and source are credited. 
up the oxidative balance. In the presence of oxidative stress, i.e. of a positive oxidative balance, the most vulnerable system of a body is the central nervous system. Health state analysis has thus shifted from cell level to molecular level (molecular biology-FR) and to atomic level (atomic physics-electrons). This is the biochemical and biophysical context in which the action of anti-oxidative compounds, taking into account that oxidative stress is responsible for about $80-90 \%$ of chronic-degenerative diseases associated to old age [4].

Living organisms and, particularly, the encephalus, are very sensitive to the damage produced by FR. Most probably, this is due to the following factors: electron-rich neuronal biomolecules recognize the oxygen radical, an electrophil ROS (it alters histaminergic, serotoninergic systems); neuronal membranes are rich in PUFA (privileged molecular substratum of FR); neuronal mitochondria are represented in large numbers (mitochondria are the cell core of FR); neurons are easily subjected to dedifferentiation as a result of the attack by FR on the DNA (they lose their own genetic specialization or differentiation and turn into neuter cells, with no specific function); neuronal oxygen is certainly present since the brain, unlike other organs and tissues, has an overall aerobic energetic metabolism. At brain level, there are small amounts of enzymatic and non-enzymatic antioxidants.

Vitamin C is an exception, as it has, at brain level, a concentration 50 times higher than any other part of the organism. This is the keysubstance for the protection of the central nervous system against FR. Neurons are "perennial cells" and the cumulated lesions produced by FR on the different cell structures could, in time, degrade quantitatively and qualitatively, the neurotransmission functions and result in behavioral changes. Cell lesions occur under conditions in which the rates of ROS are formed is high or when the activity of the defense system is low, when there is unbalance between the protection systems and the generation systems of ROS [5].

\section{Action mechanisms of free radicals}

FR can initiate reversible, small-size changes due not only to the low intensity of their formation, but also to the dissipation and decomposition reactions. The factor determining the increase of the intensity of free radical formation is oxygen activation. Due to the presence of this element not only in the atmosphere but also in almost all the substances in the body, the interaction of FR with oxygen is inevitable. They attack the existing pro-oxidants (substances/ions) for which the FR have an affinity. The action of FR on pro-oxidants is neutralized by the antioxidants or by the reactions of FR. The potential of the process of peroxidation consists in the exceeding prooxidant concentration and is superior numerically to the pro-oxidant concentration [6].

The postulate of the ageing theory is based on the reactions of FR involved in the changes caused by ageing. The changes are associated with the environment, with diseases, and with the intrinsic ageing process. This theory is based on the chemical nature of the reactions of FR and on their omnipresence. The most reactive of all oxygen FR is hydroxyl, which reacts with deoxyribose and the bases of the DNA. As a result of the postulate of the ageing theory, we can draw two conclusions on the relationship between the deterioration of the DNA and maximum life span. The increase agents/processes of the rate of deterioration of the DNA and the decrease of DNA recovery can speed up the ageing process. Thus, decreasing life span and apparent deterioration speed, we can decrease proportionally the speed of the specific metabolism (the energetic nutritional supplement).

ROS are considered factors that cause cell dedifferentiation since they react with chromatin, they change the bases of the DNA, and they cause ruptures of the chain; they induce chromosomal aberrations and a long life in the species with a low rate of accumulation of chromosomal aberrations; long life species have a low rate of accumulation of age pigments, lipofuscine, because ageing rate is proportional with metabolic rate for many different species. The oxidative changes of proteins under the action of ROS can result in inactivation of membrane enzymes and proteins and can induce structural changes resulting in destabilization of cell morphology when the target of the oxidative attack is cytoskeleton elements $[7,8]$. In the case of enzymes, the effect of ROS consists in a diminution of the catalytic capacity. The size of changes is determined by the relative location of the formation site of ROS, of antioxidant systems, and of target-protein. ROS play a role in mitochondrial damage and in permanent senescence. The results of ageing show a decrease of the number of mitochondria and the fact that the organites in the aged cells undergo biochemical alterations.

Mitochondrial DNA (mtDNA) can suffer a higher oxidative damage than nuclear DNA. Ageing is caused by the damage of mtDNA and by the peroxidation of lipids by FR in the inner mitochondrial membrane. Rapidly replicated cells do not allow the attack by FR because they use low levels of oxygen. The mitochondria of short-life span species generate larger amounts of ROS than long-life span species. mtDNA damage can block mitochondrial turnover and replication, with concomitant damage of the membrane (peroxidation processes). We can say that mitochondrion that consumes about $80-90 \%$ of the oxygen of a cell for the synthesis of the adenosine triphosphate (ATP) through oxidative phosphorylation serves as a target for ROS in aged tissues [9]. UVA radiations are little absorbed by nucleic acids since their maximum of absorption is $260 \mathrm{~nm}$. UVA radiations interact with the DNA through indirect mechanisms mediated by endogenous photosensitizing compounds. The fact that the irradiation of the cells in the absence of oxygen diminishes the lethal effect of the UVA radiations shows the involvement of ROS in the interaction of UVA radiations with nucleic acids. UVA induce lesions of the DNA through the splitting of phosphodiesteric bonds at the level of a single chain and the formation of crossed bonds neuraminidase-protein and dimerization of adjacent pyrimidines.

Despite of the complexity of the ageing process, a few mechanisms influence strongly the ageing rate. This concept forms the bases of longevity determining ageing hypotheses that could be stated as follows: differentiated cells represent the primary ageing process; ROS cause the differentiation; and active mechanisms stabilize the proper state of the differentiation, as well as antioxidants represent the category of longevity determinants.

A target of the radicalic attack is membrane lipids, because of the double bonds in the structure of PUFA. The most frequent PUFA in the structure of membrane phospholipids are linoleic, linolenic, arachidonic acids [10-12]. The process of peroxidation of PUFA consists in the following steps: a free radical extracts a hydrogen atom from the allylic position, with turning the PUFA into a lipid radical; then, there is intra molecular rearrangement of the double bonds with formation 
of a conjugated diene; as a result of the reaction between the conjugated diene and the molecular oxygen, there occurs a peroxyl radical which can react with another molecule of PUFA forming lipid radicals, with the turning of the peroxyl radical into lipid hyperoxide. Alternatively, the peroxyl radical can form cyclic peroxides. Lipid peroxides, once formed, undergo splitting resulting in the propagation of the reaction chain and in ramification and decomposition reactions [13]. The decomposition of the lipid peroxides results in such compounds as alkanes (ethane, pentane), aldehydes (malonic dialdehyde, hexanal, 4hydroxinonenal), epoxy and hydroxy fatty acids. The peroxidation of membrane lipid affects the structure and functions of plasmatic and organite membranes. Thus, transmembrane potentials, ionic flows, and transmembrane transport are troubled, membrane receptors are inactivated, and signaling paths are deregulated. Through the process of lipid peroxidation, there occur membrane components changes of lipid and protein nature as a result of the reaction of some amino acids with aldehyde peroxidation products [14]. FR and oxidative stress play a role in the decrease of dysfunctions at cell level and of the different diseases at body level.

The balance between the oxidative actions of free radicals and the level of antioxidants in a body essential for life and characterizes the capacity of resistance and adaptation of a living body.

\section{References}

1. Pickrell AM, Fukui H, Moraes CT (2009) The role of cytochrome c oxidase deficiency in ROS and amyloid plaque formation. J Bioenerg Biomembr 41: 453-456.

2. Rizzo AM, Berselli P, Zava S, Montorfano G, Negroni M, et al. (2010) Endogenous antioxidants and radical scavengers. Adv Exp Med Biol 698: 5267.

3. Gustafsson AB, Gottlieb RA (2008) Heart mitochondria: gates of life and death.
Cardiovasc Res 77: 334-343

4. Akinci M, Kosova F, Cetin B, Sepici A, Altan N, et al. (2008) Oxidant/antioxidant balance in patients with thyroid cancer. Acta Cir Bras 23: 551-554.

5. Cynshi O, Tamura K, Niki E (2010) Design, synthesis, and action of antiatherogenic antioxidants. Methods Mol Biol 610: 91-107.

6. Molyneux $P$ (2004) The use of the stable free radical diphenylpicrylhydrazyl (DPPH) for estimating antioxidant activity. Songklanakarin. Journal of Science \& Technology 26: 211-219.

7. Casado MF, Cecchini AL, Simão AN, Oliveira RD, Cecchini R (2007) Free radical-mediated pre-hemolytic injury in human red blood cells subjected to lead acetate as evaluated by chemiluminescence. Food Chem Toxicol 45: 945 952.

8. Galloway E, Shin T, Huber N, Eismann T, Kuboki S, et al. (2008) Activation of hepatocytes by extracellular heat shock protein 72. Am J Physiol Cell Physiol 295: C514-C520.

9. Ito $\mathrm{H}$ (2006) No-reflow phenomenon and prognosis in patients with acute myocardial infarction. Nat Clin Pract Cardiovasc Med 3: 499-506.

10. Milne GL, Seal JR, Havrilla CM, Wijtmans M, Porter NA (2005) Identification and analysis of products formed from phospholipids in the free radical oxidation of human low density lipoproteins. J Lipid Res 46: 307-319.

11. Wolin MS (2009) Reactive oxygen species and the control of vascular function Am J Physiol Heart Circ Physiol 296: H539-H549.

12. Deger Y, Dede S, Belge A, Mert N, Kahraman T, et al. (2003) Effects of X-ray radiation on lipid peroxidation and antioxidant systems in rabbits treated with antioxidant compounds. Biol Trace Elem Res 94: 149-156.

13. Kaynar H, Meral M, Turhan H, Keles M, Celik G, et al. (2005) Glutathione peroxidase, glutathione-S-transferase, catalase, xanthine oxidase, $\mathrm{Cu}$ $\mathrm{Zn}$ superoxide dismutase activities, total glutathione, nitric oxide, and malondialdehyde levels in erythrocytes of patients with small cell and non-small cell lung cancer. Cancer Lett 227: 133-139.

14. Sowers JR (2002) Hypertension, angiotensin II and oxidative stress. N Engl J Med 346: 1999-2001. 